\title{
On-chip stimulated Brillouin scattering and its applications
}

Benjamin J. Eggleton, Christopher G. Poulton, David Marpaung, Blair Morrison, Irina V. Kabakova, et al.

Benjamin J. Eggleton, Christopher G. Poulton, David Marpaung, Blair Morrison, Irina V. Kabakova, Duk-Yong Choi, Steve J. Madden, Barry LutherDavies, Ravi Pant, "On-chip stimulated Brillouin scattering and its applications," Proc. SPIE 8808, Active Photonic Materials V, 88081B (11 September 2013); doi: 10.1117/12.2025231

SPIE Event: SPIE NanoScience + Engineering, 2013, San Diego, California, United States 


\title{
On-chip stimulated Brillouin scattering and its applications
}

\author{
Benjamin J. Eggleton, ${ }^{(1)}$ Christopher G. Poulton, ${ }^{(2)}$ David Marpaung, ${ }^{(1)}$ Blair Morrison, ${ }^{(1)}$ \\ Irina V. Kabakova, ${ }^{(1)}$ Duk-Yong Choi, ${ }^{(3)}$ Steve J. Madden, ${ }^{(3)}$ Barry Luther-Davies, ${ }^{(3)}$ \\ and Ravi Pant ${ }^{(1)}$ \\ ${ }^{(1)}$ Centre for Ultrahigh bandwidth Devices for Optical Systems (CUDOS), Institute of \\ Photonics and Optical Science (IPOS), School of Physics, The University of Sydney, NSW \\ 2006, Australia. \\ ${ }^{(2)}$ CUDOS, University of Technology Sydney, NSW 2007, Australia \\ ${ }^{(3)}$ CUDOS, Laser Physics Centre, Australian National University, Canberra, ACT 0200, \\ Australia
}

\begin{abstract}
We review recent demonstration of stimulated Brillouin scattering in a chalcogenide photonic chip and its application to optical and microwave signal processing tasks. The interaction between light and sound via stimulated Brillouin scattering (SBS) was exploited in chalcogenide photonic circuits to achieve on-chip SBS slow and fast light, microwave photonic filters, and dynamic gratings using travelling-wave geometry. Using a ring-resonator geometry, photonic-chip based Brillouin laser was demonstrated.
\end{abstract}

Keywords: stimulated Brillouin scattering, on-chip, chalcogenide, slow-light, dynamic grating, microwave signal processing, dynamic grating, Brillouin laser

\section{INTRODUCTION}

Stimulated Brillouin scattering is a nonlinear scattering process where a pump wave $\left(\omega_{p}\right)$, when encounter acoustic vibrations $\left(\Omega_{B}\right)$ in the medium, gets backscattered, resulting in a light beam which is down-shifted in frequency $\left(\omega_{s}=\omega_{p}-\Omega_{B}\right)$ with respect to the pump by the acoustic frequency [1]. This backscattered signal is known as the Stokes wave. A probe, centered at the Stokes frequency, experiences gain when counter-propagated to the pump wave whereas a probe that is up-shifted with respect to the pump $\left(\omega_{a s}=\omega_{P}+\Omega_{B}\right)$, known as the anti-Stokes wave, experiences absorption. The gain and absorption associated with the Stokes and anti-Stokes frequency exhibit a resonant behavior, where the resonance has a Lorentzian shape whose linewidth $\left(\Delta v_{B}\right)$ is determined by the acoustic phonon lifetime in the medium. The long phonon lifetime, which is typically of-the-order of several nanoseconds in most of the commonly used optical materials, results in a narrow SBS linewidth $\sim 10 \mathrm{MHz}$. The narrow gain and absorption response of the SBS process has recently been exploited in long $(\sim \mathrm{km})$ silica optical fibers for applications in optical and microwave signal processing. The small Brillouin scattering cross-section $\left(g_{B}\right)$ and large effective mode area $\left(A_{e f f}\right)$ of silica fiber necessitated the use of long lengths $(L \sim \mathrm{km})$ in order to obtain large gain $G=$ $g_{B} P_{p} L A_{\text {eff }}$ using reasonable pump power $\mathrm{P}_{\mathrm{p}} \sim 100 \mathrm{~mW}$. Exploiting SBS in a chip-scale device is critical to realize photonic integration of optical and microwave signal processing tasks and Brillouin lasers. However, this requires generating large gain $(G)$ in short length using moderate power level, which is challenging due to the constraints on the optical and acoustic properties of material and device.

Here we review our recent work on exciting SBS in a chalcogenide photonic-chip [2-4] and its application to a number of signal processing tasks such as slow- and fast-light induced tunable delay [5], Brillouin dynamic gratings [6], microwave photonic filters [7-9] and Brillouin laser [10]. The key to these demonstrations was the large Brillouin scattering cross-section $\left(g_{B} \sim 0.74 \times 10^{-9} \mathrm{~m} / \mathrm{W}\right)$ and small mode area $\left(A_{\text {eff }} \sim 2.3 \mu \mathrm{m}^{2}\right)$ of the photonic chip [4], which resulted in large gain at low powers. Slow- and fast-light will be discussed in Section 2 followed by on-chip dynamic gratings in Section 3. In Section 4, we discuss the application of narrow SBS gain/loss profile to

Active Photonic Materials V, edited by Ganapathi S. Subramania, Stavroula Foteinopoulou, Proc. of SPIE Vol. 8808, 88081B - @ 2013 SPIE · CCC code: 0277-786X/13/\$18 - doi: 10.1117/12.2025231 
microwave photonic filter followed by review of photonic-chip Brillouin laser in Section 5. In Section 6, we present a summary of our results followed by conclusion.

\section{ON-CHIP SBS SLOW AND FAST LIGHT}

SBS based slow and fast light exploit the gain and absorption resonances, where the large index slope, resulting as a consequence of the Kramers-Kronig relation, leads to significant reduction (advancement) in the speed of light pulses centered at the Stokes (anti-Stokes) frequencies [11-14]. In order to realize SBS slow-light in short lengths $(\sim \mathrm{cm})$, careful consideration must be given to optical and acoustic properties of the material and device because the slowing down factor $\left(\Delta n_{g}=g_{B} P_{p} c / \Gamma_{B} A_{e f f}\right)$ depends on their parameters, where the Brillouin linewidth $\Gamma_{B}$ is determined by the phonon lifetime in the material and $P_{p}$ is the pump power. Large $\Delta n_{g}$ required to achieve large delay $\left(T_{d}=L \Delta n_{g} / c\right)$ in short length using small powers, therefore, can be achieved using a device with large $g_{B}$ and small $A_{\text {eff. }}$ A material with large refractive index $(n)$ and elasto-optic coefficient $\left(p_{12}\right)$ when used to design a device that provides strong acousto-optic overlap $(\eta)$ results in large $g_{B}$, which is given according to:

$$
g_{B}=\eta \frac{4 \pi n^{8} p_{12}^{2}}{c \rho v_{B} \Delta v_{B} \lambda_{p}^{3}},
$$

where material parameters such as density $(\rho)$, Brillouin shift $v_{B}$ and linewidth $\Delta v_{B}=\Gamma_{B} / 2 \pi$ typically vary with in a factor of two for commonly used optical materials. Strong dependence of $g_{B}$ on material parameters such as $n$ and $p_{12}$ however plays a crucial role in material choice. For example, silica $(n=1.445)$ and chalcogenide $(n=2.45)$ have nearly the same $p_{12}$ however; the large $n$ of chalcogenide glass results in a $\frac{n_{A s_{2} S_{3}}^{8}}{n_{\text {Silica }}^{8}} \sim 70$, which is a significant increase in $g_{B}$ just based on the choice of material.

In a recent demonstration, SBS slow- and fast-light was reported in a $7 \mathrm{~cm}$ long chalcogenide photonic-chip where the large refractive index $(n)$ and elasto-optic coefficient $\left(p_{12}\right)$ when combined with the strong acousto-optic overlap $(\eta)$ in the chip (see Fig. 1) resulted in large $g_{B}$. The large $g_{B}$ and small $A_{\text {eff }}$ of the photonic-chip generated large $\Delta n_{g}$, required for chip-based SBS slow-light. The Brillouin shift and gain bandwidth for the photonic chip were $7.7 \mathrm{GHz}$ and $34 \mathrm{MHz}$ respectively. The chip was used to measure $\Delta n_{g}$ (see Fig. 2(a, b)) and pulse delay (see Fig. 2(c, d)), which can be tuned by varying the pump power. The $\Delta n_{g}$ for the slow (fast) light regime, obtained from the delay (advancement) of a low frequency sinusoidal signal, was found to be 68 (-44) using a gain (absorption) of $11 \mathrm{~dB}(-7 \mathrm{~dB})$. Tunable delays ranging from $-7 \mathrm{~ns}$ to $23 \mathrm{~ns}$ were measured for $25 \mathrm{~ns}$ and $100 \mathrm{~ns}$ long nearly
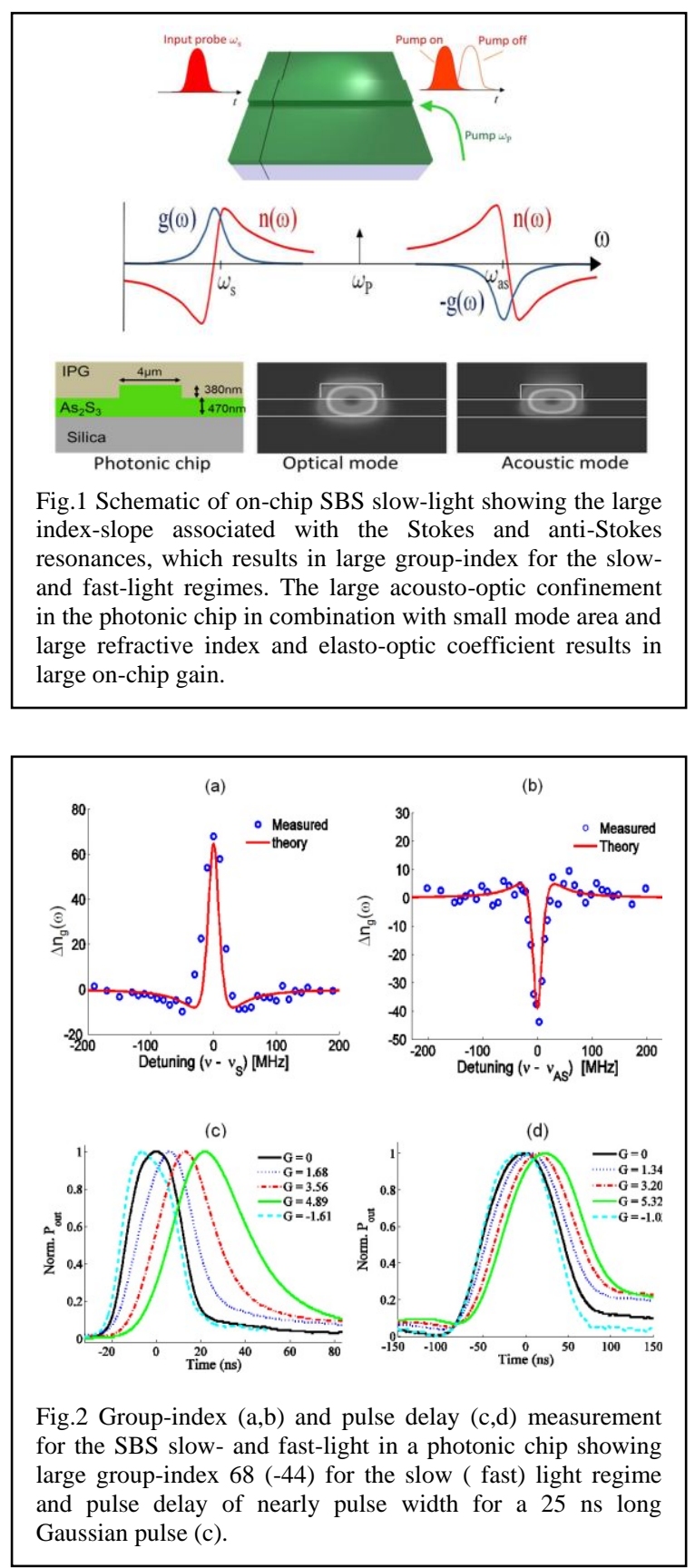

Gaussian pulses. A large group-index change $\Delta \mathrm{n}_{\mathrm{g}} \sim 130$ was obtained from the delay of $100 \mathrm{~ns}$ pulse when a gain of $23 \mathrm{~dB}$ 
was generated at a pump power of $\sim 300 \mathrm{~mW}$. This $\Delta \mathrm{n}_{\mathrm{g}}$ is nearly 13 times larger than the record $\Delta \mathrm{n}_{\mathrm{g}} \sim 10$ for the fiber-based SBS slow-light, which was obtained when a pump power of $\sim 650 \mathrm{~mW}$ was injected in a $2 \mathrm{~m}$ long erbium-doped tellurite fiber to realize a gain of 53dB [15]. SBS in a chalcogenide photonic-chip, therefore, provides a highly efficient, compact device for realizing large tunable delays. This large tunable delay can be used for applications in phased array antenna and for designing microwave photonic filters.

\section{PHOTONIC-CHIP BASED MICROWAVE PHOTONIC FILTER}

Narrowband, tunable and reconfigurable microwave photonic filters (MPFs) with high Q-factor are critical to microwave signal processing. Reconfigurable, narrowband MPFs with wide tuning range have been demonstrated using SBS in long length $(\sim 10-20 \mathrm{~km})$ optical fibers [16], which are incompatible with photonic integration. On-chip MPFs have been demonstrated using ring resonators; however demonstrated filter $3 \mathrm{~dB}$ bandwidth $\left(\mathrm{f}_{3 \mathrm{~dB}} \sim 1 \mathrm{GHz}\right.$ ) and small tuning range has resulted in very low $\mathrm{Q}$-factor $\sim 10$.

Recently, an on-chip narrowband, reconfigurable, microwave photonic filter with large $\mathrm{Q} \sim 520$ and wide tuning range was demonstrated exploiting the large gain and narrow spectral response of the SBS process in a $6.5 \mathrm{~cm}$ long chalcogenide rib waveguide. The MPF maintained a stable $3 \mathrm{~dB}$ bandwidth of $23 \pm 2 \mathrm{MHz}$ (see Fig. 3(b)) and amplitude of $20 \pm 2 \mathrm{~dB}$ (see Fig. 3(b)) over a large frequency tuning range of 2-12 GHz (See Fig. 3(a)). By tailoring the pump spectrum, the 3dB bandwidth $\mathrm{f}_{3 \mathrm{~dB}}$ of the MPF was reconfigured from $\sim 20 \mathrm{MHz}$ to $\sim 40 \mathrm{MHz}$ resulting in a tuning of the shape factor $\mathrm{S}$, defined as the ratio of $20 \mathrm{~dB}$ bandwidth to $3 \mathrm{~dB}$ bandwidth $\left(S=\mathrm{f}_{20 \mathrm{~dB}} / \mathrm{f}_{3 \mathrm{~dB}}\right)$, from 3.5 to $2[7,8]$.

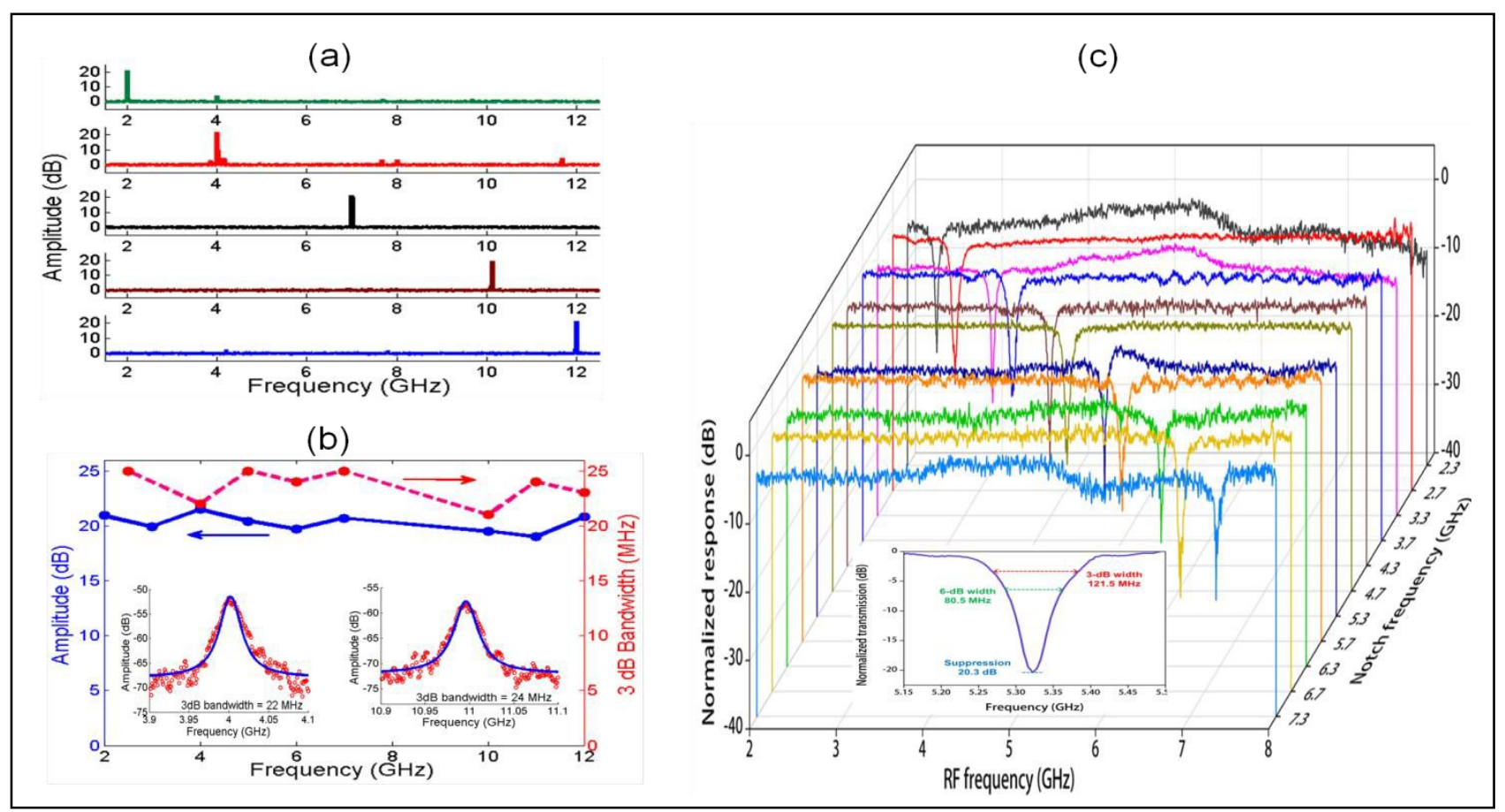

Fig.3. Measured response of the photonic-chip based microwave photonic filter showing (a) a tuning range of 2-12 GHz for a bandpass photonic filter (b) amplitude and $\mathrm{f}_{3 \mathrm{~dB}}$ for the photonic-chip based bandpass photonic filter demonstrating good amplitude $(\sim 20 \pm 2 \mathrm{~dB})$ and bandwidth $(23 \pm 2 \mathrm{MHz})$ stability $[7,8]$ and (c) tuning response of a SBS based on-chip notch filter demonstrating a wide tuning range 2-8 GHz with a $3 \mathrm{~dB}$ bandwidth of $\sim 130 \pm$ $10 \mathrm{MHz}$ and a notch depth of $\sim 18 \pm 2 \mathrm{~dB}$ [9]. Inset (b): MPF profiles taken at centre frequencies of 4 and $11 \mathrm{GHz}$ and (c) notch response at 53.GHz.

As mentioned earlier, SBS process generates gain and absorption resonances at the Stokes and anti-Stokes frequency. Using the SBS absorption in a chalcogenide photonic-chip, a widely tunable (2-8 GHz), microwave photonic notch filter (see 
Fig. 3(c)) was demonstrated with a $3 \mathrm{~dB}$ notch bandwidth of $\sim 130 \pm 10 \mathrm{MHz}$ and a notch depth of $\sim 18 \pm 2 \mathrm{~dB}$ [9]. This demonstration enables on-chip microwave signal processing using SBS.

\section{ON-CHIP BRILLOUIN DYNAMIC GRATING}

Dynamic gratings provide a novel platform for applications such as sensing, microwave signal processing and tunable delay $[17,18]$. Recently Brillouin dynamic gratings (BDGs) have been demonstrated in tens-of-meter long polarization maintaining fibres (PMFs)[19], in single-mode fibers (SMFs) [20], and in few-mode fibres (FMFs) [21]. Photonic integration of functionalities such as microwave signal processing, tunable delay lines and data storage requires realization of on-chip BDG. Realization of Brillouin grating in a photonic-chip, however, has been a challenge due to the small cross-section of the stimulated Brillouin scattering (SBS) process in silica glass, which is the commonly used material for optical fibers.

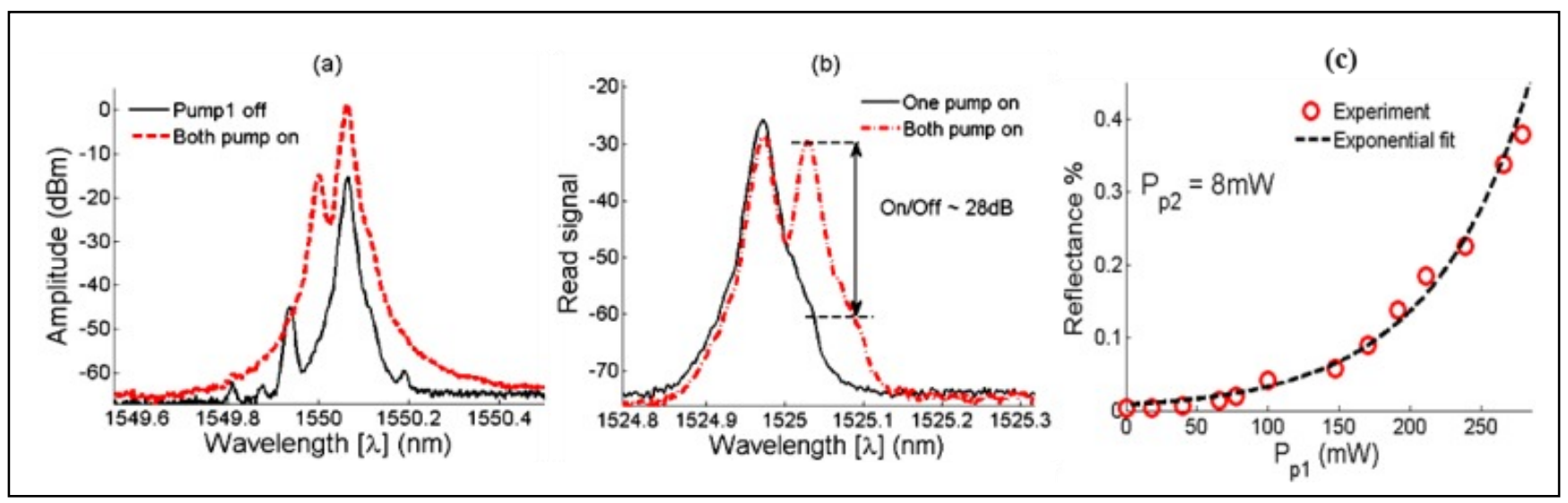

Fig. 4 (a) Pump2 spectrum when pump1 is off (solid) and on (dashed) demonstrating Brillouin gain and generation of BDG; (b) switching characteristics of the BDG when both pumps are turned on resulting in an On/Off ratio $\sim 28 \mathrm{~dB}$ and (c) reflectance of the on-chip BDG for powers $\mathrm{P}_{\mathrm{p} 1}$ of pump 1 [6].

Recently, dynamic gratings were demonstrated using large gain and high birefringence of the chalcogenide photonic-chip discussed in previous sections. Figure 4 shows the switching characteristic and reflectance of an on-chip Brillouin dynamic grating. When the pump1 is off (on), pump2 experiences no gain (gain: see dashed Fig. 4(a)) demonstrating the generation of a BDG; where both pump1 and pump2 are x-polarized. The BDG is then characterized by launching a probe in $y$ polarization. When the pump1 in x-polarization is off, no read signal is observed in the backscattered signal (solid Fig. 4(b)) [6]. However, when both the pumps are turned on a large read signal with an On-Off ratio $~ 28 \mathrm{~dB}$ was observed (dashed Fig. 4(b)). The reflectance of BDG for different pump1 power $\left(\mathrm{P}_{\mathrm{p} 1}\right)$ while pump2 power $\left(\mathrm{P}_{\mathrm{p} 2}=8 \mathrm{~mW}\right)$ is fixed is shown in Fig. 4(c) demonstrating an exponential growth, as expected because Brillouin gain increases exponentially with $\mathrm{P}_{\mathrm{p} 1}$. All the applications of on-chip SBS discussed so far rely on the travelling-wave geometry where both the pump and the probe propagate the device once. It is well-known that in the presence of feedback, the power required to excite SBS reduces significantly, where the threshold reduction depends on the quality factor $\mathrm{Q}$ of the resonator, and results in lasing action. In the next section, we discuss application of SBS in chalcogenide photonic-chip to realize a Brillouin laser.

\section{PHOTONIC-CHIP BASED BRILLOUIN LASER}

Brillouin lasers have been known for their narrow linewidth, low noise and low threshold properties. Recent focus on realizing efficient, low noise sources for coherent optical communications and microwave photonic signal processing has generated an interest in on-chip Brillouin lasers. Low threshold, low-frequency noise Brillouin lasers were recently demonstrated using silica wedge resonators, where both the pump and Stokes were aligned with the cavity resonances [22, 23], and used for realizing a highly stable, on-chip microwave source. Here we discuss a different approach where only the Stokes signal experiences feedback [24] and use it to realize a photonic-chip based Brillouin laser. 
Figure 5 shows the principle of the Brillouin laser based on a chalcogenide photonic chip where on-chip tapers were used to improve coupling efficiency [25]. In this configuration (see Fig. 5(a)), pump propagates through the chip only once before it is removed by a circulator whereas the Stokes signal recirculates in a ring comprising a $7 \mathrm{~cm}$ long photonic chip and $10 \mathrm{~m}$ long silica fibre with chip acting as a gain medium. The laser demonstrated a threshold of $360 \mathrm{~mW}$ [10], which is nearly five times smaller than that for the single-pass geometry, and a slope efficiency of $30 \%$ (see Fig. 5(b)). In this demonstration, the measured linewidth of $100 \mathrm{kHz}$ was 15 times smaller than the linewidth $(1.55 \mathrm{MHz})$ of the Brillouin pump and 340 times smaller than the Brillouin bandwidth (34 MHz) in the photonic-chip [4] .

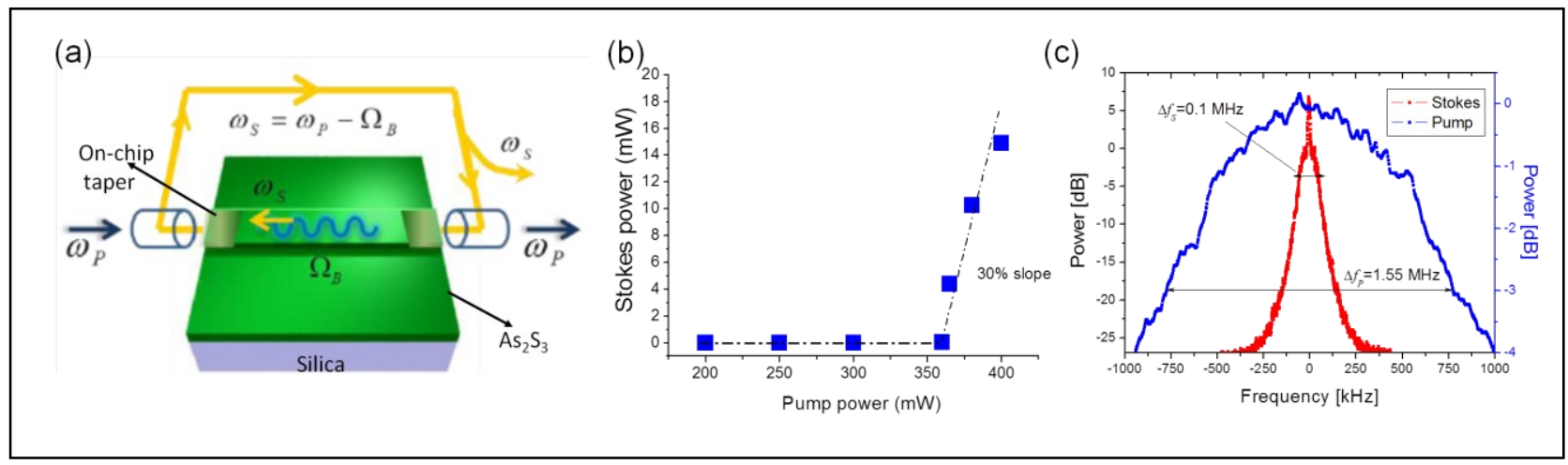

Figure 5 (a) Schematic showing the principle of photonic-chip based Brillouin laser and its threshold characteristics (b) and linewidth measurement (c) [10].

The demonstration of on-chip SBS using travelling-wave and resonator geometry has opened up opportunities for optical and microwave signal processing. New methods of fabricating chalcogenide photonic chips have recently been explored to harness SBS in a chip platform [26]. While chalcogenide photonic chip provides large on-chip SBS gain, realizing SBS in a silicon nanostructure would result in giant SBS enhancement due to combined effect of radiation pressure and electrostriction [27]. Harnessing SBS in a CMOS compatible platform will enable mass production of photonic integrated circuits for signal processing, nanolasers and tailoring the interaction between light and sound [28, 29].

\section{CONCLUSION}

We discussed our recent demonstration of realizing slow, fast and negative group velocities using on-chip SBS, where $\Delta \mathrm{n}_{\mathrm{g}}$ was continuously varied from -44 to +130 resulting in group velocities of $2307 \mathrm{~km} / \mathrm{s}$ and $-6818 \mathrm{~km} / \mathrm{s}$ for slow- and fast-light respectively. Such a wide variation allowed continuously tunable, on-chip delays ranging from $-6 \mathrm{~ns}$ to $+23 \mathrm{~ns}$ in a $7 \mathrm{~cm}$ long photonic chip with the maximum delay achieved at the relatively low pump power of $\sim 300 \mathrm{~mW}$. On-chip Brillouin dynamic gratings and microwave photonic filters with record performance were presented. A photonic-chip based Brillouin ring laser demonstrated a 15 times reduction in the laser linewidth. In future, application of chirped dynamic grating for efficient pulse storage and retrieval will be explored [30]. Fully integrated Brillouin laser using distributed feedback will provide a stable low noise source for coherent optical communication [31].

\section{ACKNOWLEDGEMENTS}

This work was funded by the Australian Research Council (ARC) through its Discovery grant (DP1096838), Laureate Fellowship (FL120100029), Center of Excellence CUDOS (Grant \# CE110001018) and Future Fellowship ( FT110100853). We acknowledge the contribution of Dr. Enbang Li.

\section{REFERENCES}

1. $\quad$ Boyd, R.W., Nonlinear Optics. 2003, Academic Press. 
2. $\quad$ Eggleton, B.J., B. Luther-Davies, and K. Richardson, Chalcogenide photonics. Nature Photonics, 2011. 5(3): p. 141-148.

3. $\quad$ Eggleton, B.J., et al., Photonic chip based ultrafast optical processing based on high nonlinearity dispersion engineered chalcogenide waveguides. Laser \& Photonics Reviews, 2012. 6(1): p. 97-114.

4. $\quad$ Pant, R., et al., On-chip stimulated Brillouin scattering. Optics Express, 2011. 19(9): p. 8285-8290.

5. Pant, R., et al., Photonic-chip-based tunable slow and fast light via stimulated Brillouin scattering. Opt. Lett., 2012. 37(5): p. 969-971.

6. $\quad$ Pant, R., et al., Observation of Brillouin dynamic grating in a photonic chip. Opt. Lett., 2013. 38(3): p. 305-307.

7. Byrnes, A., et al., Photonic chip based tunable and reconfigurable narrowband microwave photonic filter using stimulated Brillouin scattering. Opt. Express, 2012. 20(17): p. 18836-18845.

8. Pant, R., et al. Photonic chip based tunable and dynamically reconfigurable microwave photonic filter using stimulated Brillouin scattering. in Postdeadline Advanced photonics congress, Colorado Springs. 2012: Optical Society of America.

9. Marpaung, D., et al. Microwave Photonic Notch Filter using On-Chip Stimulated Brillouin Scatteringchip based tunable and dynamically reconfigurable microwave photonic filter using stimulated Brillouin scattering. in CLEO pacific rim. 2013: Optical Society of America.

10. Kabakova, I.V., et al., Narrow linewidth Brillouin laser based on chalcogenide photonic chip. Optics Letters, 2013. In Press.

11. Boyd, R.W. and D.J. Gauthier, Controlling the Velocity of Light Pulses. Science, 2009. 326(5956): p. 1074-1077.

12. Gonzalez-Herraez, M., K.Y. Song, and L. Thevenaz, Optically controlled slow and fast light in optical fibers using stimulated Brillouin scattering. Applied Physics Letters, 2005. 87(8).

13. Okawachi, Y., et al., Tunable all-optical delays via Brillouin slow light in an optical fiber. Physical Review Letters, 2005. 94(15).

14. Thevenaz, L., Slow and fast light in optical fibres. Nature Photonics, 2008. 2(8): p. 474-481.

15. Abedin, K.S., G.W. Lu, and T. Miyazaki, Slow light generation in singlemode Er-doped tellurite fibre. Electronics Letters, 2008. 44(1): p. 16-U21.

16. Zhang, W.W. and R.A. Minasian, Widely Tunable Single-Passband Microwave Photonic Filter Based on Stimulated Brillouin Scattering. Ieee Photonics Technology Letters, 2011. 23(23): p. 1775-1777.

17. Thevenaz, L., et al. Dynamic Brillouin gratings: A new tool in fibers for all-optical signal processing. in Photonics Conference (PHO), 2011 IEEE. 2011.

18. Santagiustina, M., et al., All-optical signal processing using dynamic Brillouin gratings. Sci. Rep., 2013. 3.

19. Song, K.Y., et al., All-optical dynamic grating generation based on Brillouin scattering in polarization-maintaining fiber. Optics Letters, 2008. 33(9): p. 926-928.

20. Song, K.Y., Operation of Brillouin dynamic grating in single-mode optical fibers. Optics Letters, 2011. 36(23): p. 4686-4688.

21. Li, S., M.-J. Li, and R.S. Vodhanel, All-optical Brillouin dynamic grating generation in few-mode optical fiber. Opt. Lett., 2012. 37(22): p. 4660-4662.

22. Lee, H., et al., Chemically etched ultrahigh-Q wedge-resonator on a silicon chip. Nat Photon, 2012. 6(6): p. 369373.

23. Li, J., H. Lee, and K.J. Vahala, Microwave synthesizer using an on-chip Brillouin oscillator. Nat Commun, 2013. 4.

24. Tow, K.H., et al., Brillouin fiber laser using As38Se62 suspended-core chalcogenide fiber, in Micro-Structured and Specialty Optical Fibres, K. Kalli and A. Mendez, Editors. 2012.

25. Madden, S., et al., Low loss coupling to sub-micron thick rib and nanowire waveguides by vertical tapering. Opt. Express, 2013. 21(3): p. 3582-3594.

26. Levy, S., et al., Stimulated Brillouin scattering amplification in centimeter-long directly written chalcogenide waveguides. Opt. Lett., 2012. 37(24): p. 5112-5114.

27. Rakich, P.T., et al., Giant Enhancement of Stimulated Brillouin Scattering in the Subwavelength Limit. Physical Review X, 2012. 2(1): p. 011008.

28. Rakich, P.T., P. Davids, and Z. Wang, Tailoring optical forces in waveguides through radiation pressure and electrostrictive forces. Optics Express, 2010. 18(14): p. 14439-14453. 
29. Shin, H., et al., Tailorable stimulated Brillouin scattering in nanoscale silicon waveguides. Nat Commun, 2013.4.

30. Winful, H.G., Chirped Brillouin dynamic gratings for storing and compressing light. Opt. Express, 2013. 21(8): p. 10039-10047.

31. Winful, H.G., I.V. Kabakova, and B.J. Eggleton, Model for distributed feedback Brillouin lasers. Opt. Express, 2013. 21(13): p. 16191-16199. 